\title{
Is There Evidence That Primary Care Physician Supply Influences Mammography Use?
}

Goals for the health of our communities and nation have been set for the first decade of this millennium. ${ }^{1}$ The Surgeon General led an effort that defined 467 objectives in 28 focus areas and established an ambitious agenda for health care. That agenda includes three objectives: (1) increase the proportion of persons with a regular primary care provider from $77 \%$ to $85 \%^{1}$; (2) increase the proportion of women aged 40 years and older who have had a mammogram in the previous 2 years from $68 \%$ to $70 \%^{1}$; and (3) decrease breast cancer mortality in women from 27.7 to 22.2 in $100,000{ }^{1}$

In this issue of the $\mathcal{A} A B F P$, Ferrante and colleagues $^{2}$ report a cross-sectional analysis of incident breast cancers during 1994. They link the cancer data with physician data and estimate the association between primary care physician supply and the odds of early-stage cancer. They suggest that women residing in a Florida county with a higher proportion of primary care providers have increased odds of early breast cancer detection and conclude that increasing the supply of primary care physicians could improve breast cancer outcomes.

This interesting study raises some important methodological challenges. Its findings are based on a cross-sectional analysis, and the authors appropriately recognize that the associations could be due to other unmeasured factors. This study would be strengthened by a clearer conceptualization of what those factors might be. A lack of conceptualization of factors influencing mammography makes it more difficult to compare studies and learn from each. When weak associations are found, the confounding variables that are not measured become even more important. This study shows weak associations between primary care supply and earlystage cancer. Conceptualization of the problem needs to be a more fundamental part of this and other health services research so it will be possible

Submitted 16 August 2000.

From the Group Health Cooperative, Seattle, Wash. Address reprint requests to Stephen Taplin, MD, Group Health Cooperative, 1730 Minor Avenue, Suite 1600, Seattle, WA 98101. to determine the potential unmeasured variables and guide our work with time.

In my own work, ${ }^{3}$ I have used a heuristic conceptualization based upon work by Green and Kreuter, ${ }^{4}$ Bandura, ${ }^{5}$ and Fishbein and Ajzen. ${ }^{6}$ It includes measurement of both individual and environmental factors. Though Ferrante and colleagues state they have controlled for individual level factors, such as age, race, comorbidity, and health insurance, these factors are only some that might influence mammography use. Other individual level variables include beliefs, values, past preventive behavior, perceived risk, and perceptions of what others in the environment want them to do (ie, social support). None of these variables could be measured in the reported study, so the confounding of the associations by these factors remains a concern. The framework I use also considers environmental factors, such as health care system support and logistics. Health care system support includes coverage of the tests and reminder systems for patients or their physicians. Logistics include the ease of appointment making, the distance to a center, and the details of transportation.

The analysis by Ferrante et al is based upon the importance of considering the environment within which individual persons operate. This issue is critical, and there is now a growing interest in how the environment influences behavior beyond what might be measured at the individual level. ${ }^{7}$ The influence of the environment is important to behavioral as well as etiologic research, but it is hard to address in research implementation. How much does living in a community discussing breast cancer prevention influence early-stage detection beyond what the individual person perceives? A person might perceive a stronger social norm, but that measurement alone might not account for the community effect. A person could belong to a health maintenance organization (HMO), but the penetration of HMOs might also influence providers and care beyond the level of individual patients.

The fundamental issue addressed by including environmental measures in an analysis is finding 
appropriate measures at the community level. How does one measure the effect of an HMO upon care within the community at large? That challenge is greater in the work by Ferrante and colleagues because they use administrative databases. These databases provided few relevant environmental measures, though they are a rich resource for health care service use, costs, and cancer outcomes. For example, were any promotional campaigns underway in the counties that might influence social norms and explain the differences? Were there incentives for the primary care providers that might not be present for specialty providers? Were reminder systems incorporated into primary care that were not part of specialty care? Were facilities closer to the patient population in some counties? Environmental factors can indeed be more important than individual level variables. ${ }^{8}$ Physician workforce mix might be among those environmental factors that need closer evaluation as an influence upon outcomes, but it is not the only factor, and administrative data sets alone are unlikely to provide the array of measures needed.

The analysis provided here suggests that total physician supply is not associated with increased early detection, though the association does exist for primary care physician supply. Even if the individual level factors were measured without confounding, one has to wonder why primary care physician supply might influence outcomes. There has been considerable concern that primary care physicians are not providing recommendations to obtain screening. ${ }^{9}$ If the association is real, then we need to understand what primary care physicians do differently from specialty physicians, teach it, and promote primary care physician supply by stating clearly why that supply improves outcomes.

Although I might be cautious about the associations between primary care physician supply and breast cancer outcomes shown by Ferrante and colleagues, the importance of their work could be that they are evaluating the associations at all. They have conducted a similar analysis of colon cancer screening and found an important association with primary care physician supply. ${ }^{10}$ Developing an analytic approach to how physician supply influences outcomes is critical to improving the health of the nation. The Healtby People 2010 goals highlight the need for primary care physicians to guide individual patient care. Since 1990 there has been a total increase in physicians by 117,245 but a drop in the proportion of family physicians and general internists from $26.8 \%$ in 1990 to $25.9 \%$ in $1997 .{ }^{11}$ During the late 1980s and early 1990s, there was considerable discussion of physician workforce and the risk of a physician glut. ${ }^{12,}{ }^{13}$ Little recent analysis is available to determine whether these fears have been realized. More importantly, the need to evaluate the effect of supply upon outcomes is much greater.

How supply influences outcomes is important to family physicians but more important to our patients. The Healthy People 2010 goals set primary care access targets because it is believed that a link exists between access and outcomes. Ferrante and colleagues begin to provide a basis for that belief, but much more research must be done to show that the link is real. Ultimately our patients are those who need to know our practice makes a difference, and it is their mortality that our practice should influence. Family physician survival, like family practice, should be based upon evidence, not dogma. We must show that what we do influences outcomes, if it does. Ferrante and colleagues have begun to develop methods that would help us do such work, but much more is yet to be done before the evidence is clear.

Stephen Taplin MD, MPH Seattle, Wash

\section{References}

1. Shalala DE. Healthy people 2010. Conference edition Vol I and II. Colllingdale: DIANE Publishing, 2000:1-5, 7-23, 24-46.

2. Ferrante JM, Gonzalez EC, Pal N, Roetzheim RG. Effects of physician supply on the early detection of breast cancer. J Am Board Fam Pract 2000;13:xxx-xx.

3. Taplin SH, Barlow WE, Ludman E, et al. Testing reminder and motivational telephone calls to increase screening mammography: a randomized study. J Natl Cancer Inst 2000; 92:233-42.

4. Green LW, Kreuter MW. Health promotion planning: an educational and environmental approach. In: Health promotion today and a framework for planning. Mountain View, Calif: Mayfield Publishing, 1991:24.

5. Bandura A. The self system in reciprocal determinism. Am Psychol 1978; 33:344-58.

6. Fishbein M, Ajzen I. Determinants of behavioral intentions. In: Understanding attitudes and predicting social behavior. Paramus, NJ: Prentice-Hall, 1980:53-60. 
7. Diez-Roux AV. Bringing context back into epidemiology: variables and fallacies in multilevel analysis. Am J Public Health 1998; 88:216-22.

8. McBride CM, Curry SJ, Taplin SH, Anderman C, Grothaus L. Exploring environmental barriers to participation in mammography screening in an HMO. Cancer Epidemiol Biomarkers Prev 1993;2: $599-605$.

9. Screening mammography: a missed clinical opportunity? Results of the NCI Breast Cancer Screening Consortium and National Health Interview Survey Studies. JAMA 1990;264:54-8.
10. Roetzheim RG, Pal N, Gonzalez EC, et al. The effects of physician supply on the early detection of colorectal cancer. J Fam Pract 1999;48:850-8.

11. Health, United States 1999 with health and aging chartbook. Washington, DC: US Government Printing Office, 1999:269, Table 1.

12. Schwartz WB, Mendelson DN. No evidence of an emerging physician surplus: an analysis of change in physicians' work load and income. JAMA 1990;263: 557-60.

13. Cooper RA. Seeking a balanced physician workforce for the 21 st century. JAMA 1994; 27:680-7. 